\title{
Rare Presentation of Caries Exudata with Dumbbell-Shaped Abscess in an Adult - A Case Report
}

\author{
M Kishore Kumar ${ }^{1}$, Sameer Panchal ${ }^{2}$, N Sabarinathan ${ }^{3}$, Akil Prabhakar ${ }^{3}$
}

\section{Learning Point of the Article:}

The aim of this case report is to give an ideation about the presentation of caries exudata in adults, a rare fulminating form of glenohumeral joint tuberculosis and despite of such malignant clinical presentation, the condition can be managed well with conservative treatment if diagnosed precisely with appropriate clinical examination, radiological, and microbiological investigations.

\section{Abstract}

Introduction: Tuberculosis (TB) of the shoulder joint is a sparse entity. Here, we are presenting a case of glenohumeral joint TB (caries exudata variant) with 1-year follow-up.

Case Presentation: Our patient, a 65-year-old male individual, came to the outpatient department with complaints of swelling and pain in the right shoulder of 6 months duration. Patient's systemic examination and vitals were within normal limits, and local examination of the shoulder joint revealed massive dumbbell-shaped swelling, tenderness along the joint line, and grossly restricted range of movements. Radiograph of the shoulder joint showed osteolytic lesions in the humeral head and glenoid with a reduction in glenohumeral joint space. We treated the case with the aspiration of the abscess followed by three sessions of injection streptomycin into the cavity at monthly intervals. The patient also received anti-tubercular drugs as per the Revised National Tuberculosis Control Program guidelines for 1 year. After 12 months, the patient was diseasefree and had a functional range of motion.

Conclusion: In adults, the classical dry type of shoulder TB (caries sicca) has been described, while the fulminating variety rarely has been reported. The latter type of shoulder TB is much more common in children. We report a fulminant variety of shoulder joint TB in an old-aged patient who presented with massive dumbbell-shaped cold abscess and destruction of glenohumeral joint.

Keywords: caries sicca, caries exudate, glenohumeral joint, injection streptomycin

\section{Introduction}

The incidence of tuberculosis (TB) of the shoulder joint is $1-2.8 \%$ of the skeletal TB $[1,11]$. The dry type of lesion is commonly reported in adults, while the fulminating variety of shoulder TB is common in children $[3,8]$. We report a 65 -yearold patient with the right glenohumeral joint TB in whom the clinical presentation was of the acute fulminating type and simulated a sarcomatous lesion with severe destruction of the shoulderjoint.

\section{Case Report}

department on March 2019 with complaints of pain in the right shoulder for the past 6 months and dumbbell-shaped swelling progressively increasing in size around the same region for the past 4 months. He had severe pain and global restriction of movements in the right shoulder which urged him for medical attention. There was no history of trauma, manipulation, fever, or night sweats. The patient had a history of pulmonary TB 2 years back, for which he was treated with anti-tubercular drugs according to the Revised National Tuberculosis Control Program (India) guidelines for 6 months and declared sputum negative. There is no history of any other systemic disorders.

A 65-year-old male presented to us in the outpatient

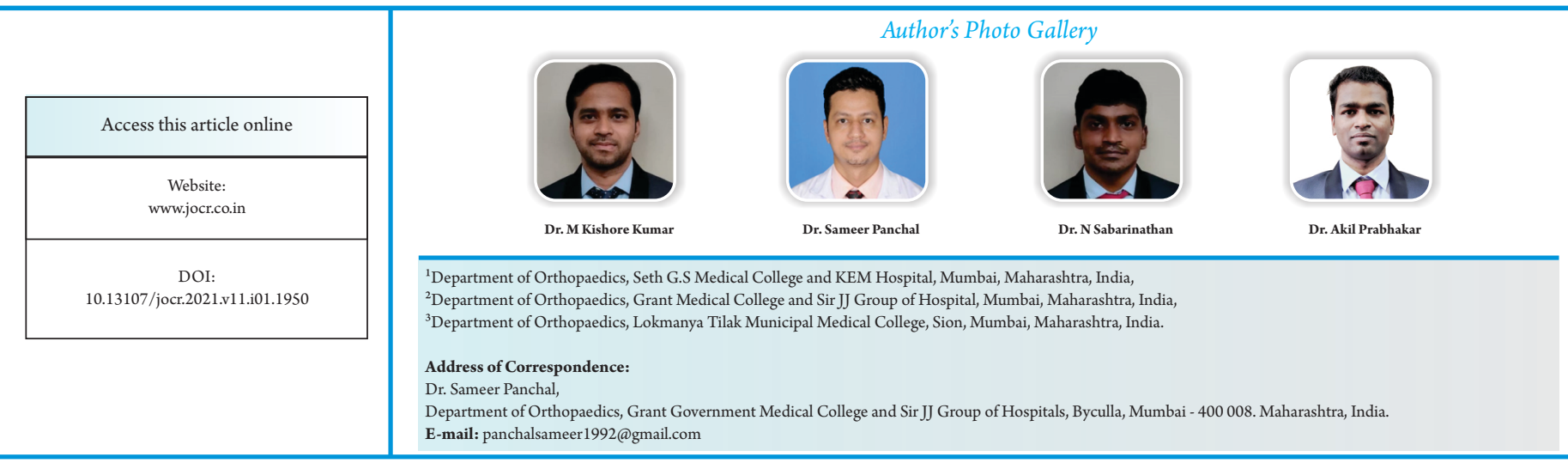

Journal of Orthopaedic Case Reports | pISSN 2250-0685 | eISSN 2321-3817 | Available on www.jocr.co.in | doi:10.13107/jocr.2021.v11.i01.1950 This is an Open Access article distributed under the terms of the Creative Commons Attribution Non-Commercial License (http://creativecommons.org/licenses/by-nc/3.0) which permits unrestricted non-commercial use, distribution, and reproduction in any medium, provided the original work is properly cited. 


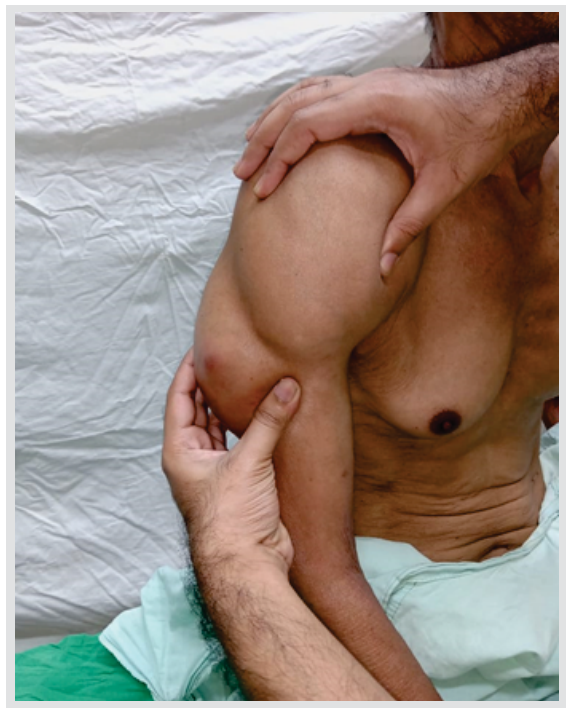

Figure 1: Image showing a clinical picture of the right shoulder with massive swelling demonstrating fluctuation. Clinical image of the right shoulder showing a massive dumbbell-shaped swelling, soft in consistency with positive fluctuation.

\section{Local examination}

Reveals massive dumbbell-shaped swelling over the right shoulder which is soft in consistency with ill-defined margins showing cross-fluctuation (Fig. 1-3). The overlying skin was tense and shiny, with no venous engorgement. There was no discharging sinus or pointing abscess. Local temperature was normal. Tenderness was present on palpation of anterior and posterior capsule region, deep axilla, and lateral aspect of the scapula. Movements of the shoulder were painful and restricted in all directions. There was no distal neurovascular deficit. Axillarylymph nodes were enlarged.

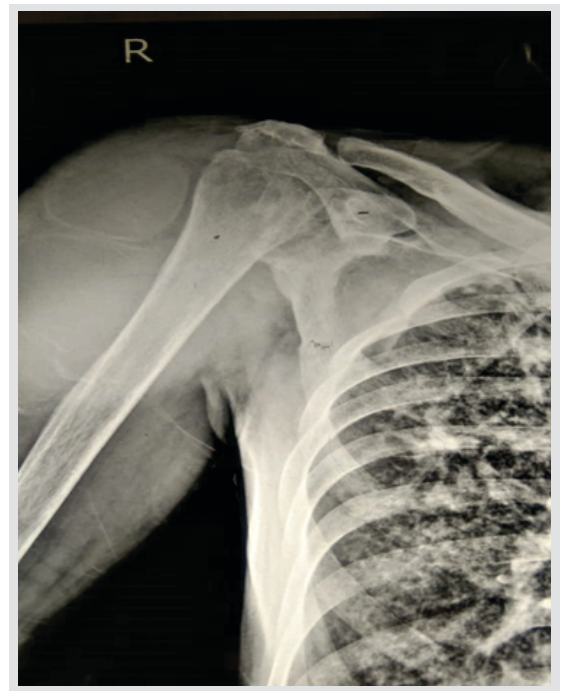

Figure 4: Anteroposterior radiograph of the right shoulder at the time of presentation. Plain radiograph of the right shoulder anteroposterior view shows erosion of the glenoid cavity and head of the humerus with a reduction in the join space. Obliteration of adjacent fat planes is seen with minimal erosion of lateral end of clavicle.

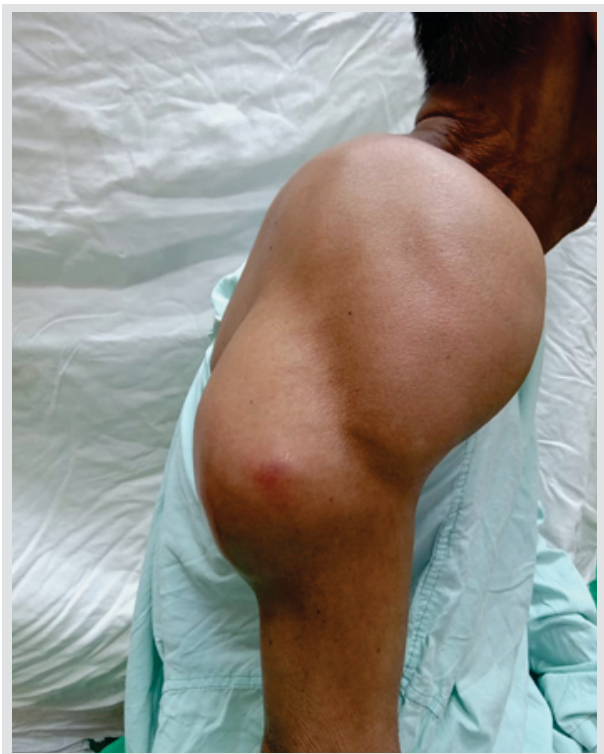

Figure 2: Image showing lateral aspect of the right shoulder with dumbbell-shaped swelling. Clinical image of the right shoulder showing lateral aspect of dumbbell-shaped swelling.

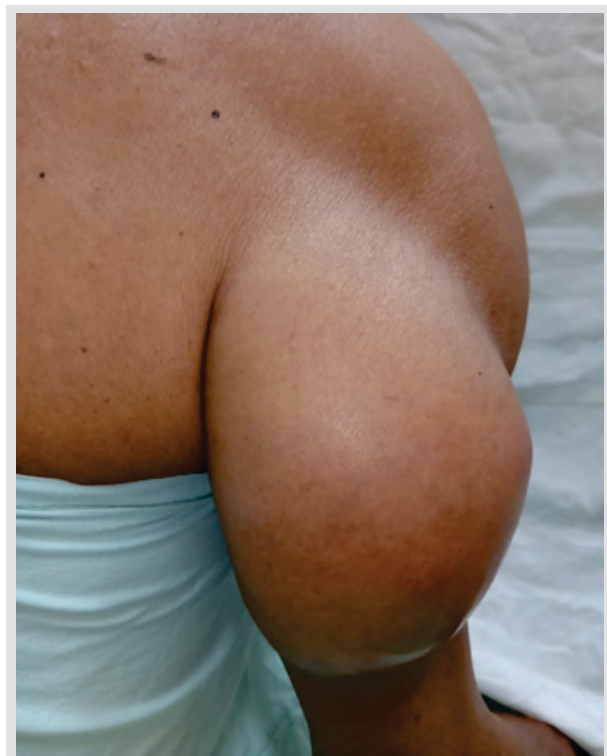

Figure 3: Image showing posterior aspect of the right arm. Clinical image of the right shoulder showing posterior aspect of dumbbellshaped swelling.

\section{Blood investigations}

Revealed hemoglobin $(\mathrm{Hb})$ of $11 \mathrm{gm} \%$, a total leucocyte count of $11,000 \mathrm{~mm} 3$, differential count of $48 \%$ lymphocytes, $50 \%$ polymorphs, $2 \%$ eosinophils, and erythrocyte sedimentation rate (ESR Westergren) was $60 \mathrm{~mm} / \mathrm{hr}$.

\section{Radiological examination}

X-ray right shoulder anteroposterior view (Fig. 4) shows erosion of the glenoid cavity and head of the humerus with a reduction in the joint space. Obliteration of adjacent fat planes is seen with minimal erosion of lateral end of the clavicle. Softtissue shadow is suggestive of gross fluid collection. There is no dislocation of the glenohumeral joint.

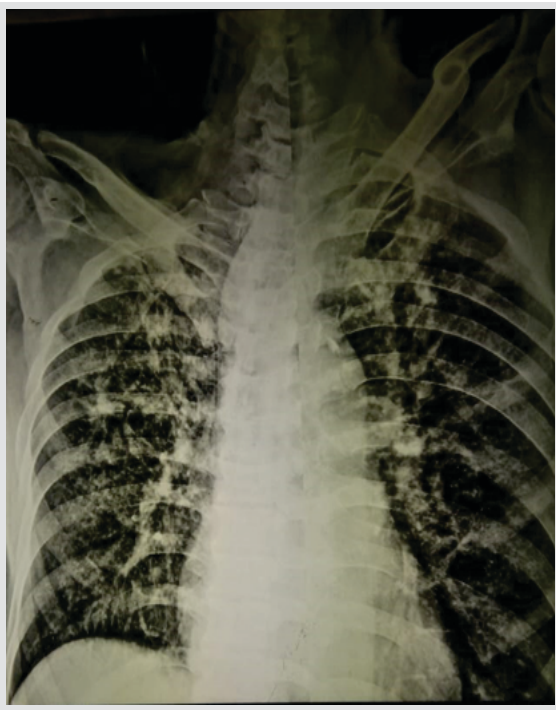

Figure 5: Chest radiograph showing miliary changes in both the lung fields. Plain posteroanterior radiograph of chest showing widespread small nodular opacities distributed throughout the lung fields suggestive of miliary tuberculosis (pasthistory)

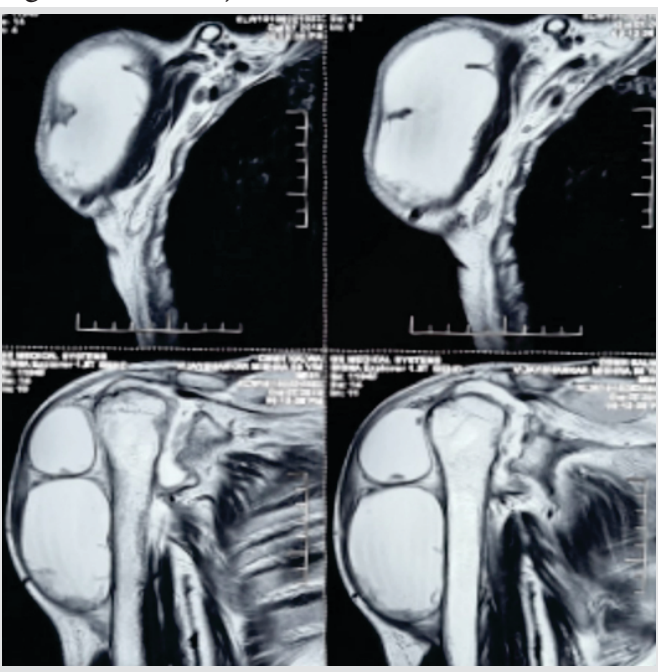

Figure 6: Magnetic resonance imaging T2-weighted coronal section. Magnetic resonance imaging of the right shoulder T2-weighted coronal cut showing cortical erosions involving articular margins of humeral head and bony glenoid. There are moderate joint effusion and synovial thickening with a large loculated periarticular collection extending to supraspinatus fossa and upper arm along with the deltoid muscle. 


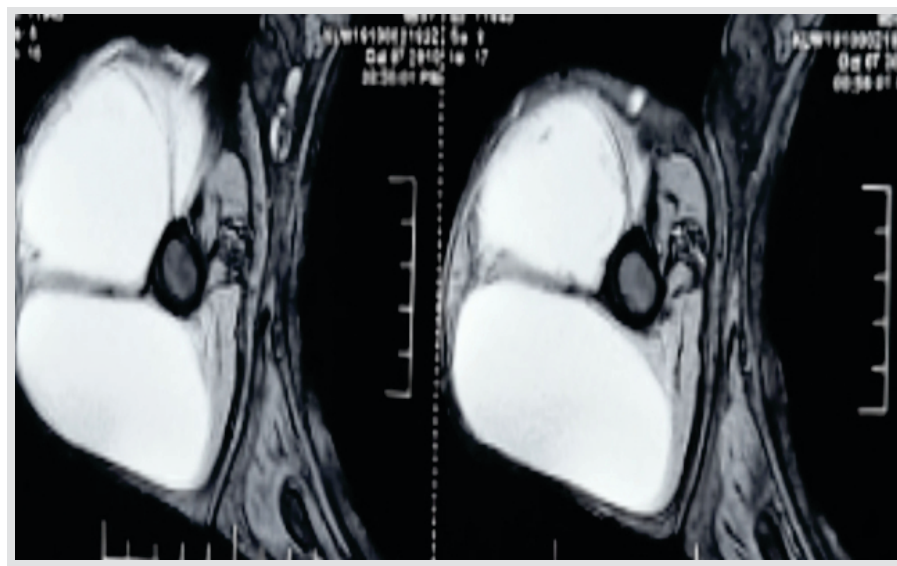

Figure 7: Magnetic resonance imaging T2-weighted axial section of the right humerus. Magnetic resonance imagin of the right shoulder $\mathrm{T} 2$-weighted axial cut showing a large bilocular collection around right humerus with soft-tissu edema.

Chest X-ray (Fig. 5) showed widespread small nodular opacities distributed throughout both the lungs suggestive of miliary TB.

Magnetic resonance imaging right shoulder joint (Fig. 6, 7) shows cortical erosions involving articular margins of the humeral head and bony glenoid. Proximal migration of humeral head at the shoulder joint is seen. There are moderate joint effusion and synovial thickening with a large loculated periarticular collection extending to supraspinatus fossa and upper arm along with the deltoid muscle. Largest collection measures approximately $14 \mathrm{~cm} \times 6 \mathrm{~cm}$. There are rotator cuff muscles atrophy and few enlarged lymph nodes in the axilla. This picture was suggestive of tuberculous arthropathy of the right shoulder joint.

Diagnostic aspiration of the cold abscess was done from the non-dependent region under aseptic precaution, material aspirated was sent for pus culture, acid-fast bacilli (AFB) staining, Gene-Xpert, and culture for Mycobacterium tuberculosis (Mtb). AFB staining came negative. MTb was detected with Gene-Xpert documenting no rifampicin resistance. Culture with Mycobacterium growth indicator tube (TB-MGIT) came positive in 3 weeks with the bacilli susceptible to all first-line anti-tubercular drugs. There was no superadded pyogenic infection being ruled out by final pus culture and sensitivity report.

The patient was started on first-line anti-tubercular therapy (ATT) isoniazid, rifampicin, pyrazinamide, and ethambutol for 2 months then switched to HRE for 6 months and HR for the next 4 months. Therapeutic aspiration of the cold abscess was done and $1 \mathrm{~g}$ of streptomycin was injected into the lesion once the diagnosis was confirmed by diagnostic aspiration. The patient was provided with shoulder immobilizer and compression bandage after the procedure. A similar procedure of injecting streptomycin was repeated thrice at monthly intervals. After 3 months, the swelling and pain were alleviated; the patient was started on physiotherapy and shoulder range of
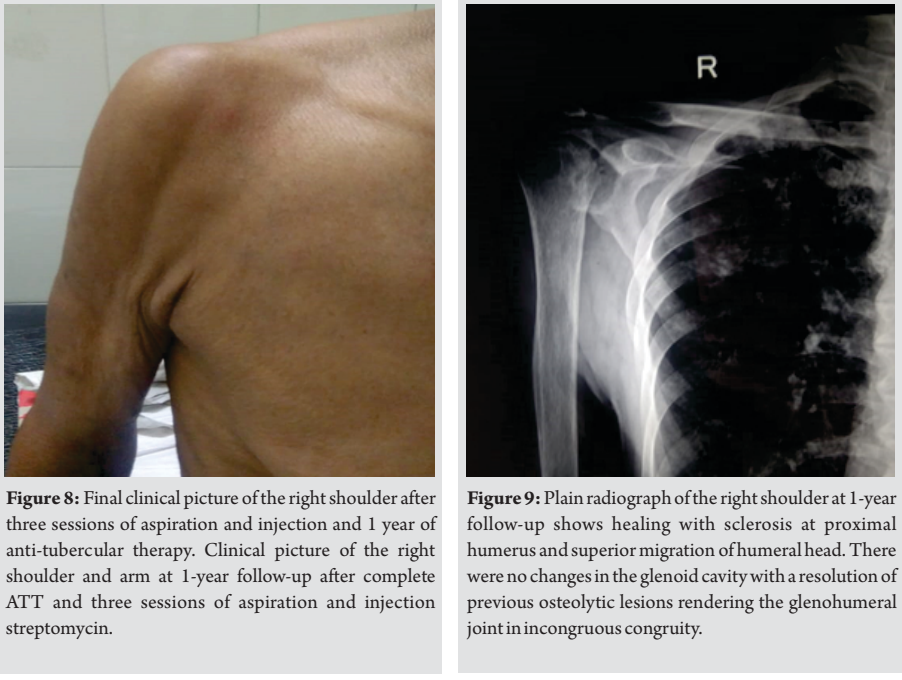

motion exercises. The total duration of multidrug antitubercular chemotherapy was 1 year. At 1 -year follow-up, although the movements were restricted (flexion $50^{\circ}$, extension $20^{\circ}$, abduction $60^{\circ}$, adduction $0^{\circ}$, internal rotation $10^{\circ}$, and external rotation $10^{\circ}$ ) and mostly scapulothoracic, the patient had gained weight, had no pain or swelling, and could carry out his day-to-day activities without much discomfort (Fig. 8). The anteroposterior radiograph (Fig. 9) showed healing with sclerosis at proximal humerus and superior migration of head of the humerus. There were no changes in the glenoid cavity.

\section{Discussion}

Osteoarticular TB is far less common than the pulmonary form. It constitutes around $1-3 \%$ of patients with extrapulmonary $\mathrm{Tb}$, with $50 \%$ of cases occurring in the spine [1]. The incidence of $\mathrm{TB}$ of the shoulder joint is rare and accounts for only $1-2.8 \%$ of cases of skeletal $\mathrm{TB}$, there is usually pulmonary involvement with skeletal TB, as was the case with our patient [1]. The infection to the joint could occur through hematogenous route or contiguous spread from an adjacent infected bone. The spread from adjacent bone infection is considered to be the most common route of transmission for osteoarticular TB [2].

TB of the shoulder joint can be classified into three types [3]:

- Type I: The classic "Caries Sicca," the dry form wherein the patient presents with marked wasting of the shoulder and painful restriction of all movements

- Type II: The “Caries Exudata," the florid type. Like in our case, the patient has a swelling of the joint, cold abscess, and occasionally a sinus

- Type III: The "Caries Mobile." This is characterized by restriction of active movements of the shoulder, but passive movements of varying degrees are present, patients having nearly full passive abduction, the diagnosis of tuberculous arthritis is generally not difficult when classic radiographic 
features appear in typical locations. With unusual features or in atypical locations, the diagnosis can be more troublesome. The appearance of periarticular osteoporosis, marginal erosions, and absent or mild joint space narrowing is most indicative of this disease.

In adults, the classical dry type of shoulder TB (caries sicca) has been described, while the fulminating variety has been sparsely reported. Tuberculous arthritis most typically affects large joints such as the knee and the hip. Rarely, other joints such as glenohumeral joint can be involved. The acute fulminating variety of disease, as seen in our patient, is reported to be extremely rare in adults but common in children and may simulate acute osteomyelitis, septic arthritis, or osteogenic sarcoma. Although our patient had symptoms related to the right shoulder for the past 6 months, he presented with acute exacerbation clinically simulating a tumorous lesion and radiologically with the destruction of the glenohumeral joint. Very few cases of such presentation of shoulder TB have been reported before in adults $\mathrm{A}$, triad of radiographic findings (Phemister's triad) is characteristic of tubercular arthritis severe periarticular osteoporosis, peripherally located osseous erosions, and gradual narrowing of the joint space [4]. In addition, there are erosions, reactive sclerosis, and periosteal reactions. Pathologic abnormalities in tuberculous arthritis include changes in synovial membrane, cartilaginous, and osseous abnormalities. An enlarging joint effusion, inflammatory thickening of the periarticular connective tissue, and fat contribute to soft-tissue swelling. The time required for loss of joint space in TB is highly variable. In some patients, diminution of this space is a late finding, occurring after marginal and central erosions of large size have appeared. In others, loss of interosseous space can be appreciated at a time when only small marginal osseous defects are apparent.

Positive Ziehl-Neelsen staining and/or a positive culture of $\mathrm{M} . \mathrm{Tb}$ from the affected bone or tissue are the gold standard for diagnosing osteoarticular TB [5]. Gene Xpert M.Tb/RIF assay is highly accurate for TB detection and offers early identification of rifampicin resistance. ESR could be high, as in our patient. However, across reported cases and case series of biopsy-proven osteoarticular $\mathrm{Tb}$, there is no consistency regarding ESR [6]. Martini et al. believed that a severe destructive tubercular lesion may not have as bad an outcome as anticipated [7]. They recommended shoulder spica immobilization in abduction and external rotation to avoid disabling stiffness in adduction. They also reported better results with conservative management as compared to arthrodesis or excision. According to Kelly and Kalson, the pathology in TB lends itself to debridement of infective tissue because the shoulder is a non-weight-bearing joint and can accept more joint irregularity [8]. Abdel Wahab et al. reported two cases of TB which mimicked neoplasms, but retrospective analysis of the radiographic features offered some clues to the more benign nature of the disease [9]. Subsequent aspiration of this cavity and cytology of the aspirate finally confirmed the lesion to be tubercular. Sahoo et al. emphasized the value of fineneedle aspiration cytology in the diagnosis of $\mathrm{TB}$ of bone and joints which mimic a neoplastic lesion [10]. Intra-articular injection of streptomycin had been used previously to treat $\mathrm{TB}$ of the knee with promising results $[11,12]$. In our case, we used it to treat glenohumeral TB and obtained an acceptable result.

\section{Conclusion}

This case brings out a very rare presentation of glenohumeral joint TB in adults with emphasis that modern imaging techniques and aspiration/culture are useful in such situations where clinical diagnosis is dubious. Caries exudata, a fulminating variant of glenohumeral $\mathrm{TB}$ being extremely rare in adults, as in our case managed with aspiration and three sessions of injection streptomycin into the cavity at a monthly interval, along with ATT for 12 months with a good functional range of motion.

\section{Clinical Message}

The aim of this case report is to give an ideation about the presentation of caries exudata in adults, a rare fulminating form of glenohumeral joint TB. Despite of such malignant clinical presentation, the condition can be managed well with conservative treatment if diagnosed precisely with appropriate clinical examination, radiological, and microbiological investigations.

\section{References}

1. Kapukaya A, Subasi M, Bukte Y, Gur A, Tuzuner T, Kilnc N. Tuberculosis of the shoulder joint. Joint Bone Spine 2006;73:177-81.

2. Tang SC, Chow SP. Tuberculosis of the shoulder-a report of 5 cases treated conservatively. J R Coll Surg Edinb 1983;28:188-90.

3. Goel V, Sharma PK, Jain J, Yadav U, Devgan A, Bhardwaj N. Caries sicca: Tuberculosis of glenohumeral joint. Int J Res 
Orthop 2019;5:747.

4. Lakhkar DL, Yadav M, Soni A, Kumar M. Unusual presentation of shoulder joint tuberculosis: A case report. Indian J Radiol Imaging 2006; 16:23-6.

5. Pais R, Maruthi CV, Vedashree MK, Buvanesh CR, Anand BP. A rare case of tuberculosis of shoulder joint: Sicca variant. Sch J Appl Med Sci 2013;1:538-41.

6. Darraj M. Delayed presentation of shoulder tuberculosis. Case Report in Infect Dis 2018;2018:8591075.

7. Martini M, Benkeddache Y, Medjani Y, Gottesman H. Tuberculosis of the upper limb joints. Int Orthop 1986; 10:17-23.

8. Kelly PJ, Kalson AG. Musculoskeletal tuberculosis. Mayo
Clin Proc 1969;44:73-80.

9. Abdelwahab IF, Kenan S, Klein M, Rabinowitz JG. Atypical skeletal tuberculosis mimicking neoplasm. $\mathrm{Br} \mathrm{J}$ Radiol 1991;64:551-5.

10. Sahoo M, Sahai K, Nayak VM. Scapulohumeral tuberculosis diagnosed by fine needle aspiration cytology. Acta Cytol 1998;42:435-6.

11. Tuli SM. Tuberculosis of the shoulder. In: Tuberculosis of the Skeletal System. 1st ed. New Delhi: Jaypee Brothers Medical Publisher Private Ltd.; 1993.

12. Ahern RT, Arden GP. Intra-articular streptomycin in tuberculosis of the knee. BrMedJ 1952;1:466-8.
Conflict of Interest: Nil

Source of Support: Nil

Consent: The authors confirm that informed consent was obtained from the patient for publication of this case report

\section{How to Cite this Article}

Kumar K, Panchal S, Sabarinathan N, Prabhakar A. Rare Presentation of Caries Exudata with Dumbbell Shaped Abscess in an Adult - A Case Report. Journal of Orthopaedic Case Reports 2021 January; 11(1):23-27. 